\title{
SPATIAL REPRESENTATION OF FIBER BRIDGING FORCES IN STRAIN-HARDENING CEMENTITIOUS COMPOSITES
}

\author{
JINGU KANG* AND JOHN E. BOLANDER ${ }^{\dagger}$ \\ *University of California, Davis \\ Davis, CA USA \\ e-mail: jgkang@ucdavis.edu \\ ${ }^{\dagger}$ University of California, Davis \\ Davis, CA USA \\ e-mail: jebolander@ucdavis.edu
}

Key words: Fiber Reinforced Concrete, Lattice Models, Strain-hardening, Multiscale Modeling

\begin{abstract}
This research involves the development of lattice models of strain-hardening cement composites (SHCC), in which the individual fibers are explicitly represented. The pullout forces of fibers are derived from micromechanical bases and distributed along the fiber embedment lengths. This spatial representation of the fiber bridging forces provides realistic representations of stress transfer between the fiber and matrix, which is essential for simulating crack openings and crack spacing in SHCC. Multiple cracking produces islands of material interconnected by fiber bridges, which places unusual demands on the solution procedure. A special event-by-event solution strategy is adopted for this reason.
\end{abstract}

\section{Introduction}

The mechanical properties of fiber reinforced cement composites depend on the properties of the cement-based matrix; the type, amount and spatial distribution of fibers; and the properties of the fiber-matrix interface. Strain-hardening cement composites (SHCC) are a special class of fiber reinforced concrete that achieves high toughness through controlled multiple cracking. The crack openings are small, typically in less than $100 \mu \mathrm{m}$, which is attractive from a durability standpoint. Such performance depends on proper selection/proportioning of the constituent materials and on the methods of production and curing.

The robustness of SHCC designs, going from the controlled laboratory environment to practical applications, is an area of concern. Nonuniform fiber dispersion, particularly in large volume applications of SHCC, may hinder the desired strain-hardening and related properties. Such research needs have motivated the development of computational models that explicitly represent fibers within the composite material [1-9]. The ability to model individual fibers, and their collective influence on composite behavior, enables: 1) direct representation of the effects of fiber dispersion; and 2) multiscale description of fiber-local behavior, as it affects composite performance.

This form of modeling is challenging on several fronts, particularly when simulating the behavior of SHCC. A large number of fibers are used in typical mixture designs. Fine discretizations are needed to resolve the closely spaced cracks that develop under loading. Furthermore, local peaks and snap-backs occur during the process of multiple cracking. Portions of the matrix may separate from the bulk material 
and be connected only by fiber bridges, which places unusual demands on the solution procedure.

Herein, lattice models of SHCC, based on the rigid-body-spring concept, are extended to meet these challenges. Firstly, the crack bridging forces are distributed along the embedment lengths of the fibers, which suppresses spurious localization of damage and avoids mesh size sensitivity [10]. The distribution of force along the embedment lengths is determined by the micromechanics of fiber pullout $[11,12]$. Secondly, clusters of fibers are assumed to pullout within an event-by-event process that enhances the stability and computational efficiency of the solution process. The modeling approach and its potential are demonstrated through comparisons with experimental results for $\mathrm{SHCC}$ under tensile loading.

\section{Lattice modeling of SHCC}

\subsection{Matrix material}

The matrix material is represented by a lattice of rigid-body-spring elements [13,14]. Element geometry and connectivity are defined by Voronoi/Delaunay dual tessellations of a set of nodal points, as shown in Fig. 1 for the planar arrangement of nodes considered herein. In the figure, $F_{n}, F_{s}$, and $F_{t}$ are the forces in normal and two tangential springs, respectively, located at the area centroid of the Voronoi facet common to nodes $i$ and $j$. Rotational springs are also present at the area centroid, but their actions are not indicated in the figure. The spring set couples the degrees of freedom of the nodes via rigid-arm links. Element stresses $\sigma_{n}, \sigma_{s}$, and $\sigma_{t}$ are obtained by dividing the respective spring forces by the facet area, $A_{i j}$. The modeling of matrix fracture is described in section 3 .

The element stiffness formulations provide an elastically homogeneous representation of the continuum under uniform modes of straining [14]. Correct representation of the Poisson effect, both globally and in an element-local sense, has recently been accomplished [15].

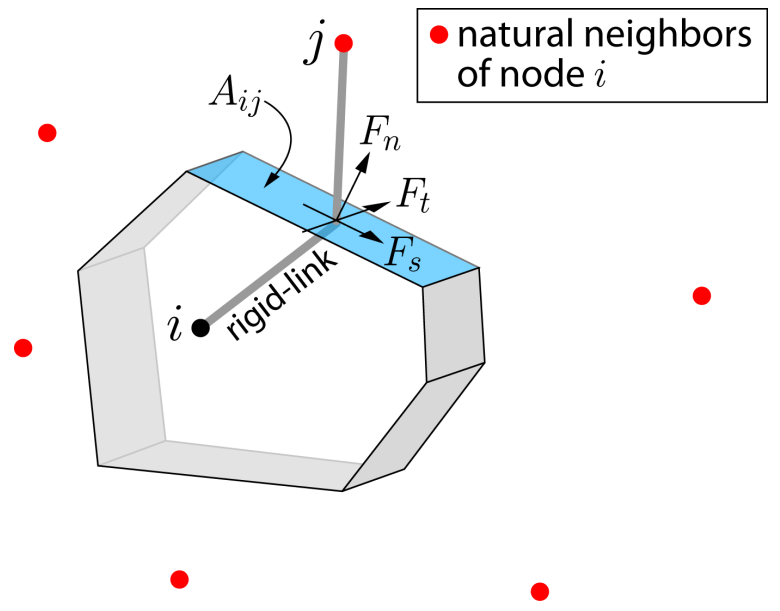

Figure 1: Lattice element $i j$ based on the rigid-bodyspring concept

\subsection{Fiber reinforcement}

The strength and stiffness contributions of each individual fiber are explicitly represented within the model, without increasing the number of system degrees of freedom [1]. A fiber element is constructed at each point of intersection of a fiber with a Voronoi facet of the background mesh representing the matrix. A zero-sized spring, oriented in the fiber direction, is placed at the intersection point. The spring is coupled to the associated nodes through rigid links, in a way that is analogous to the rigid-body-spring construction of the matrix elements.

The cracking bridging forces of fibers are commonly applied to the crack faces. This lumping of individual fiber forces, or cohesive force representation of the collective actions of fibers, is adequate for cases of single cracking. Within actual materials, however, the fiber bridging forces are transferred to the matrix along the embedment lengths of the fibers, which has a strong influence on relevant aspects of multiple cracking of SHCC, including crack spacing and opening. Furthermore, the lumped approach is not objective upon mesh refinement. With sufficiently fine mesh, application of the bridging forces directly on the crack faces causes spurious break-off of portions of the matrix [10]. 
(a)

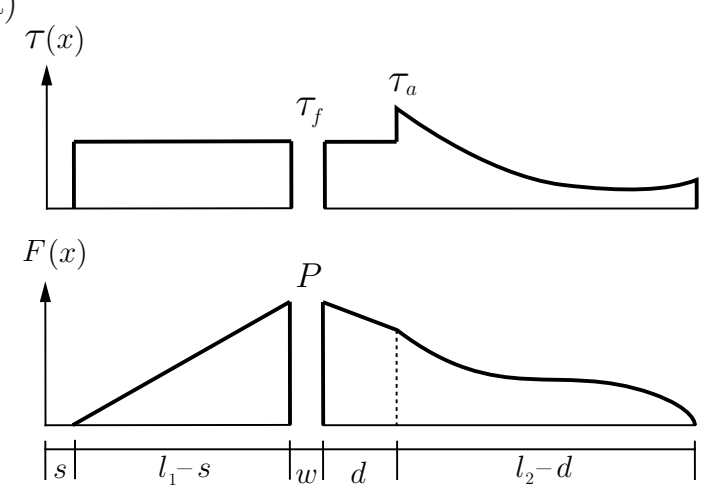

(b)

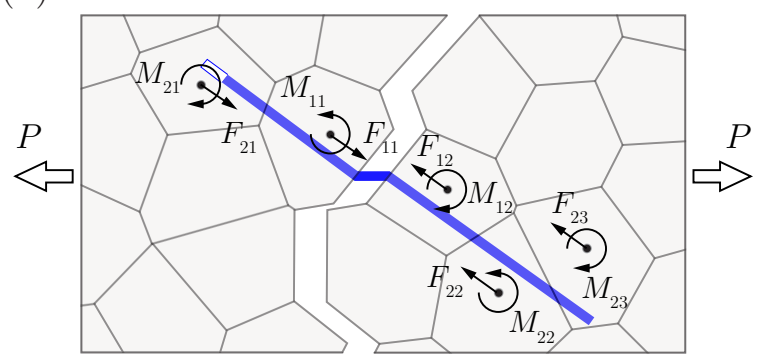

Figure 2: Distributed-force approach: (a) interfacial shear stress profiles and corresponding force distributions; and (b) distributed forces acting on associated nodes along the fiber embedment lengths to each side of a developing crack.

To address these issues, and better reflect fiber behavior within the composite material, the transfer of force from fiber to matrix is distributed along the embedment lengths of a fiber [10,12]. Figure 2 shows such a spatial, distributed force approach, where $l_{1}$ and $l_{2}$ are the fiber embedment lengths; $w$ is the crack opening; $d$ is the debonded length of fiber; $s$ is the slip at the end of the debonded fiber; $P$ is the crack bridging force; $\tau_{a}$ and $\tau_{f}$ are adhesional and frictional shear strength, respectively. The distributed forces along the embedded lengths of the fiber are based on the micromechanics of single fiber pullout [11].

The pre- and post-cracking actions of fibers depend on the movements of the Voronoi cells that represent the matrix, according to the rigidbody-spring concept. Notably, the addition of fibers does not increase the number of degrees of freedom of the system, so that large numbers of fibers can be considered without significant computational burden. Fibers are freely placed in the domain irrespective of the background discretization of the matrix. Herein, fibers are placed uniformly at random in the domain, except for the influence of domain boundaries. Alternatively, by placing fibers using spatially correlated random fields [16], it has been demonstrated that regions with fewer fibers act as potential sites for fracture localization, limiting the strain capacity of the composite material [17].

\section{Event-based solution procedure}

Event-based solution procedures have a long history within the subject area of damage modeling and are a central feature of classical lattice models [18]. The solution proceeds iteratively with one state change, or event, allowed per computational cycle. With respect to lattice models, an event is commonly the breaking or removal of an element. The critical element is selected according to a fracture criterion or some other measure of criticality. Advantages of an event-based solution include the avoidance of: 1) zero-energy modes of deformation associated with complete break-off of a portion of the domain; and 2) spurious dependence of the solution on load step size. A significant drawback is the large computational expense associated with solving the system equations for each individual state change. As the computational grid is refined, expense increases not only due to the addition of nodes, but also due to the additional events needed to produce a crack or other damage feature. (a)

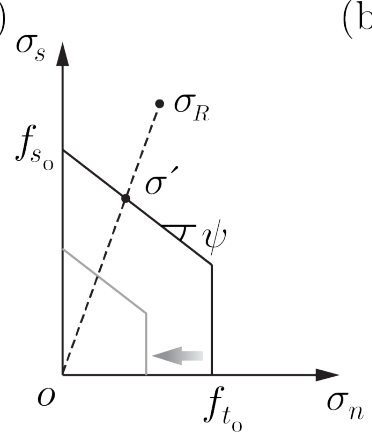

(b)

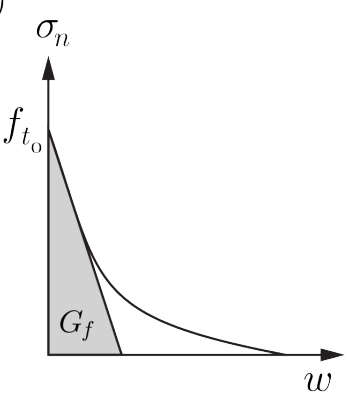

Figure 3: a) Failure criterion defined by a Mohr-Coulomb surface with a tension cut-off; and b) softening relation for uniaxial tension 


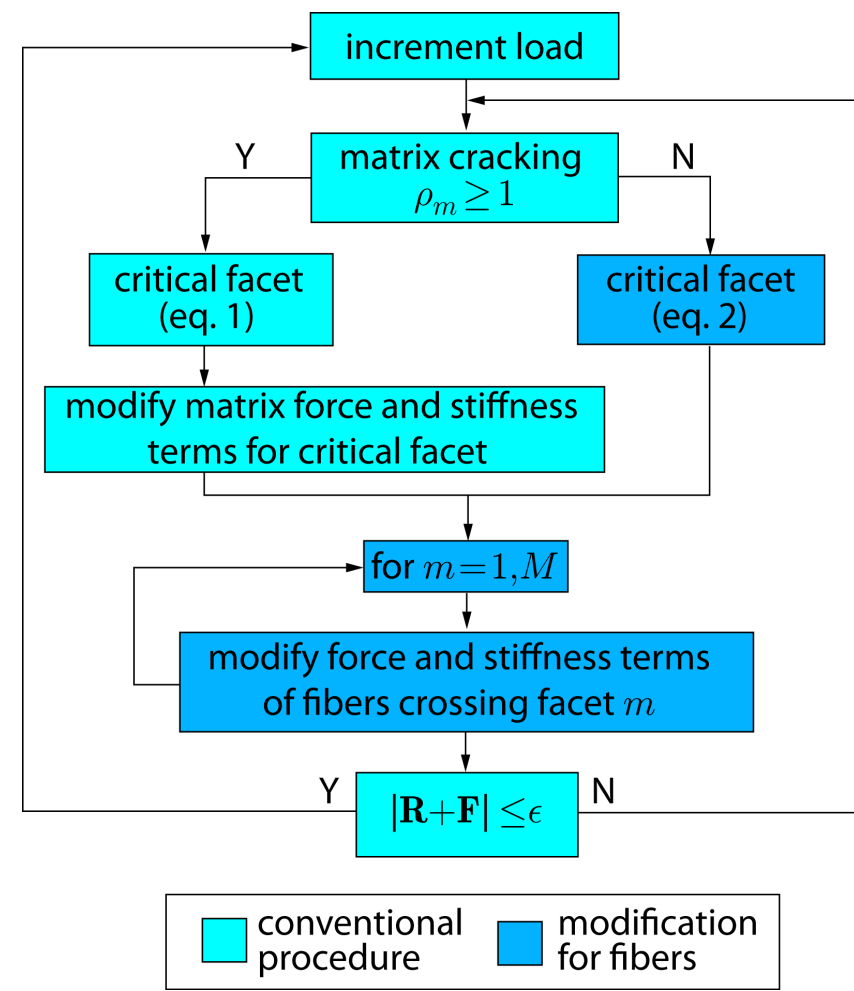

Figure 4: Event-by-event solution procedure for fiber reinforced materials

Cracking of the matrix phase is based on an established event-by-event procedure [19]. We identify the critical element as the one with the largest stress ratio

$$
\rho_{m}=\max \left[\frac{\sigma_{R}}{\sigma^{\prime}}\right]
$$

where $\sigma_{R}=\left(\sigma_{n}^{2}+\sigma_{s}^{2}+\sigma_{t}^{2}\right)^{1 / 2}$ is the resultant matrix stress acting on the facet; $\sigma^{\prime}$ is a measure of matrix strength (or residual strength in the post-peak regime) defined by the failure surface (Fig. 3a). In the figure, $f_{t 0}$ and $f_{s 0}$ are the initial strength values in tension and pure shear, respectively, and $\psi$ is the angle of cohesion. Individual elements break incrementally to simulate material softening as a function of crack opening $w$, as shown in Fig. $3 \mathrm{~b}$, where $G_{f}$ is the fracture energy of the cement-based matrix.

This form of event-by-event procedure is herein extended to account for the post-cracking effects of fiber reinforcement, as indicated by the flow chart in Fig. 4. Following common practice, iterations are performed to establish equilibrium after each load increment. Equilibrium is established when the $\ell^{2}$ norm of the sum of the external and internal nodal forces ( $\mathbf{R}$ and $\mathbf{F}$, respectively) is less than a prescribed tolerance $\epsilon$.

Figure 5 shows the crack bridging forces of individual fibers associated with matrix element $i j$. The critical facet is determined by the maximum normalized bridging force for each such nodal pair in the network.

$$
\rho_{f}=\max \left[\frac{\sum_{k=1}^{n} f_{k}}{\sum_{k=1}^{n} f_{k}^{\prime}}\right]
$$

in which $f_{k}$ is the axial force of fiber $k$ within the cluster of $n$ fibers bridging the portion of the crack represented by the facet; $f_{k}^{\prime}$ is the reference bridging force of fiber $k$, which is defined by the intersection of the theoretical forcepullout relation and the secant branch to $f_{k}$. The definition of $f_{k}^{\prime}$ is analogous to that of $\sigma^{\prime}$ used for matrix cracking (Fig. 3). The normalizing factor $\left(\sum f_{k}^{\prime}\right)$ also serves to reduce dependence of $\rho_{f}$ on the form of discretization.

The fibers crossing the critical facet typically intersect other facets, which we call sub-critical facets (Fig. 6). The fiber forces and stiffness terms associated with the one critical and $M-1$ sub-critical facets need to be updated for each pullout event associated with the fiber cluster, as indicated by the loop structure within Fig. 4 . If the bridging forces of the fibers were lumped at the crack face, as in a conventional cohesive crack approach, then $M=1$ and there would be no sub-critical facets.

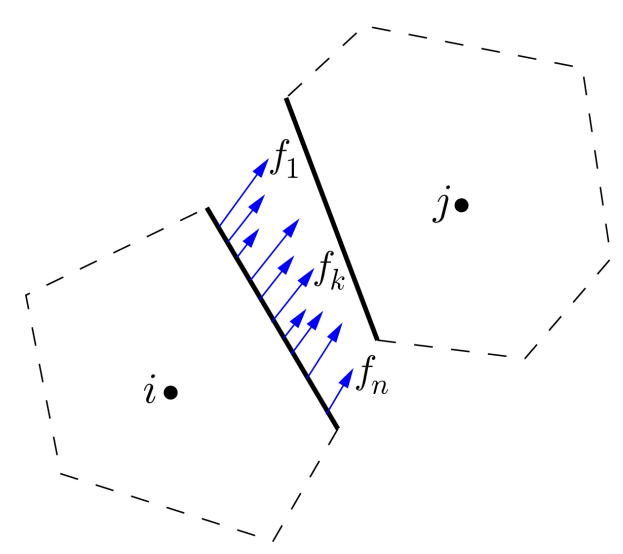

Figure 5: Cluster of bridging forces acting on the facet of cracked element $i j$ 


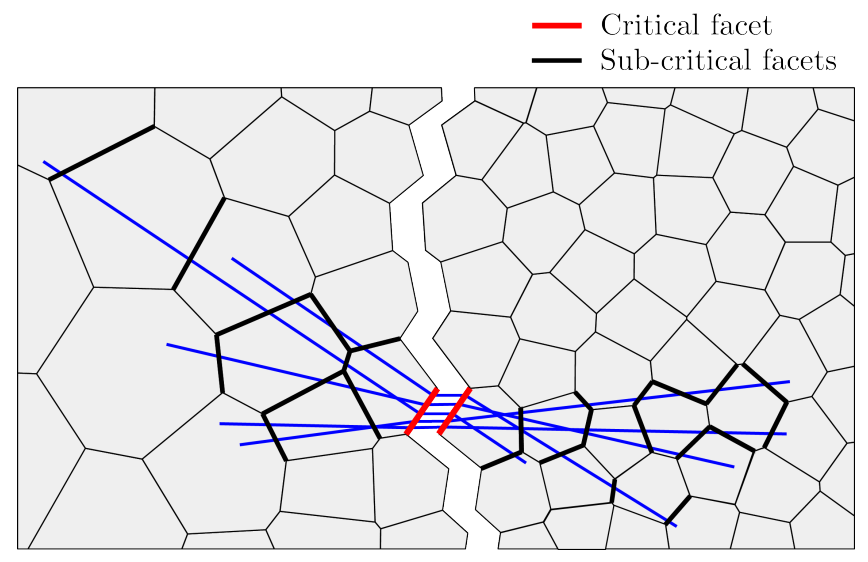

Figure 6: Critical and sub-critical facets associated with a fiber-cluster pullout event

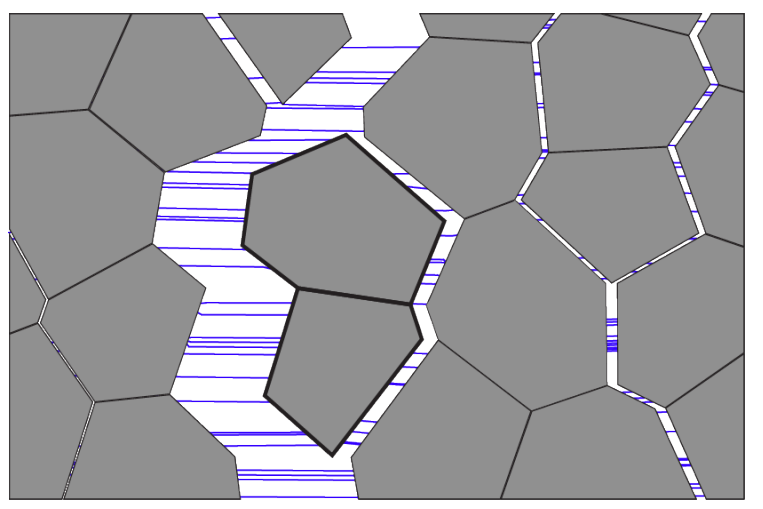

Figure 7: Matrix break-off produces an island-like feature; fibers that bridge crack faces are indicated by blue lines

Due to the presence of fibers, and the forces they carry across cracks, it is possible for portions of the matrix to become detached from the surrounding matrix. This scenario, depicted in Fig. 7, is computationally tractable using the event-based solution procedure. In contrast, the conventional solution procedure (in which fiber pullout/unloading occurs simultaneously throughout the domain within a computational cycle) fails to converge during equilibrium iterations.

\section{Simulation example}

The SHCC specimens tested by Adendorff et al. [20] are modeled by the irregular lattice, as shown in Fig. 8. In accordance with the experiments, axial loading is applied through displacement control of hinged supports. The model formulation and analyses are three-dimensional, yet a unit thickness is assumed to reduce computational expense. PVA fibers (with $12 \mathrm{~mm}$ length, $0.04 \mathrm{~mm}$ diameter, and $40 \mathrm{GPa}$ elastic modulus) are placed within the gage length at a dosage of $2 \%$ per unit volume. The assumption of unit thickness produces a planar distribution of fibers. The influence of frictional snubbing and matrix spalling on apparent bond strength $\tau$, as a function of fiber entry angle $\phi$, is modeled using the procedure given by Lee et al. [21] (Fig. 9).

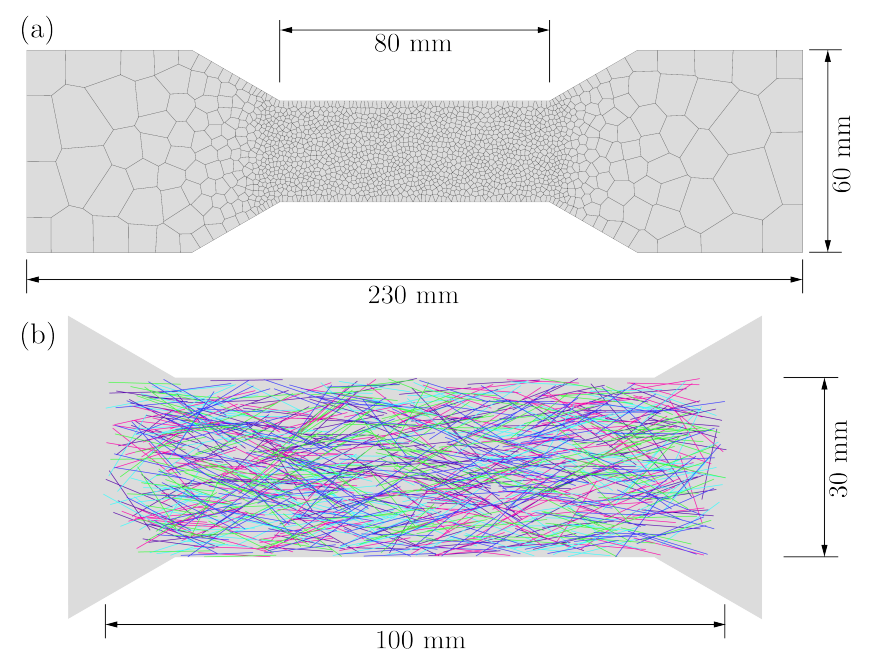

Figure 8: Modeling of SHCC specimens: a) matrix discretization; and b) short-fiber placement within the gage length

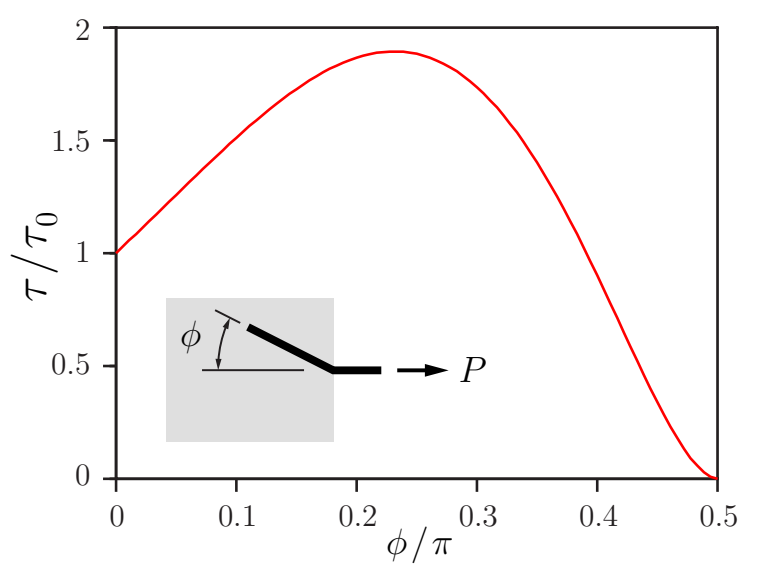

Figure 9: Relationship between apparent bond strength and fiber inclination 
As shown in Fig. 10, the lattice model results compare well with the experimentally measured stress-strain curves. In particular, the lattice model results exhibit multiple cracking and a prolonged strain-hardening branch up to fracture localization at a strain of about $4 \%$. Whereas the strengths of the matrix and fibermatrix interface were calibrated to produce this result, the similitude of the results demonstrates the potential of the model. The simulated cracking patterns for increasing levels of axial strain are presented in Fig. 11. Elsewhere, using a simpler discretization of the specimen gage length, it is shown that simulated crack count and crack opening values agree well with the experimental results [12].

These results were obtained using the aforementioned event-based solution procedure. Equilibrium iterations of a conventional solution procedure fail to converge partway into the strain-hardening regime. As a verification of the event-based solution procedure, analyses were conducted using a reduced fiber dosage of $1 \%$ for which only a single crack forms. Apart from minor differences prior to peak loading, the event-based and conventional solution procedures provide essentially the same results up through the fully cracked (traction-free) condition (Fig. 10).

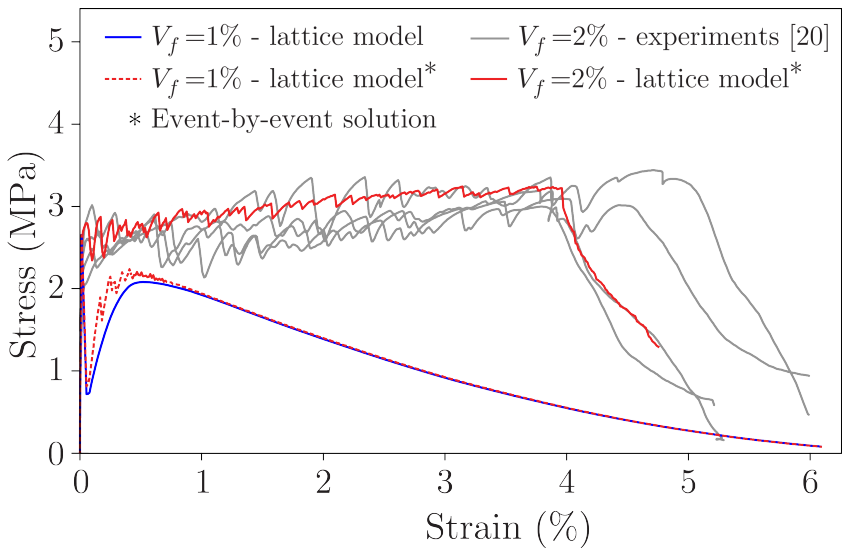

Figure 10: Comparison of simulated and experimental stress-strain responses

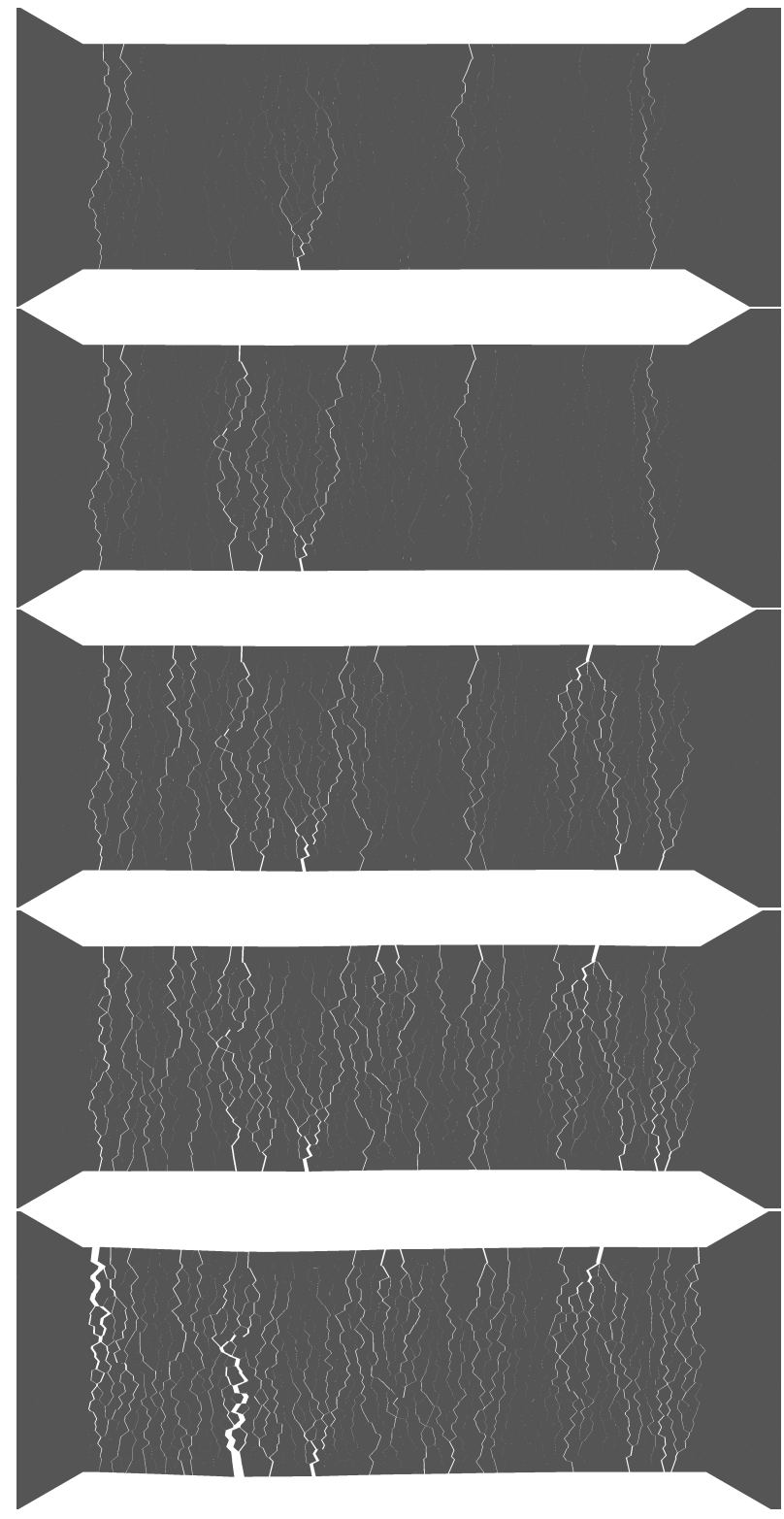

Figure 11: Simulated crack patterns using the eventbased solution technique $\left(V_{f}=2 \%\right)$; from top to bottom, images correspond to strain levels of $0.5,1.0,2.0,3.0$, and $4.0 \%$, respectively.

\section{Conclusion}

The individual and collective actions of short-fiber reinforcement in SHCC can be simulated using lattice models based on the rigidbody-spring concept. Previous work in this area has been extended in two significant ways:

1. The crack bridging forces are distributed along the embedment lengths of the individual fibers, according to micromechanical models of fiber pullout. This 
spatial representation of crack bridging forces provides realistic representations of SHCC behavior under tensile loading. The lattice model results exhibit stable formation of multiple cracks and a prolonged strain-hardening branch, up to fracture localization at large strain.

2. The proposed event-based procedure improves the stability and efficiency of the solution process. A pullout event associated with a fiber cluster involves the updating of force and stiffness terms of fibers bridging the critical facet and subcritical facets (i.e., facets traversed by the fiber embedment lengths). Whereas, a conventional solution technique fails to converge partway up the strain-hardening branch, the event-based solution procedure enables simulation of multiple cracking and the strain-hardening process up through fracture localization.

\section{REFERENCES}

[1] Bolander, J. E., and Saito, S. 1997. Discrete Modeling of Short-fiber Reinforcement in Cementitious Composites. Advanced Cement Based Materials 6:76-86. doi:10.1016/S1065-7355(97)90014-6

[2] Bolander, J. E., Choi, S., and Duddukuri, S. R. 2008. Fracture of fiber-reinforced cement composites: effects of fiber dispersion. International Journal of Fracture 154:73-86.

doi:10.1007/s10704-008-9269-4

[3] Radtke, F. K. F., Simone, A., and Sluys, L. J. 2010. A computational model for failure analysis of fibre reinforced concrete with discrete treatment of fibres. Engineering Fracture Mechanics 77:597-620. doi:10.1016/j.engfracmech.2009.11.014

[4] Cunha, V.M.C.F., Barros, J.A.O, and Sena-Cruz, J.M. 2011. An integrated approach for modelling the tensile behaviour of steel fibre reinforced self-compacting concrete. Cement and Concrete Research 41:64-76.

doi:10.1016/j.cemconres.2010.09.007

[5] Kunieda, M., Ogura, H., Ueda, N., and Nakamura, H. 2011. Tensile fracture process of Strain Hardening Cementitious Composites by means of threedimensional meso-scale analysis. Engineering Fracture Mechanics 33:956-965. doi:10.1016/j.cemconcomp.2011.05.010

[6] Schauffert, E. A., and Cusatis, G. 2012. Lattice Discrete Particle Model for FiberReinforced Concrete. I: Theory. Journal of Engineering Mechanics 138:826-833. doi:10.1061/(ASCE)EM.19437889.0000387

[7] Ellis, B.D., DiPaolo, B.P., McDowell, D.L., and Zhou, M. 2014. Experimental investigation and multiscale modeling of ultra-high-performance concrete panels subject to blast loading. International Journal of Impact Engineering 69:95103.

doi:10.1016/j.ijimpeng.2013.12.011

[8] Montero-Chacón, F., Schlangen, E., Cifuentes, H., and Medina, F. 2015. A numerical approach for the design of multiscale fibre-reinforced cementitious composites. Philosophical Magazine 95:3305-3327. doi:10.1080/14786435.2015.1040101

[9] Octávio, C., Dias-da-Costa, D., Alfaiate, J., and Júlio, E. 2016. Modelling the behaviour of steel fibre reinforced concrete using a discrete strong discontinuity approach. Engineering Fracture Mechanics 154:12-23. doi:10.1016/j.engfracmech.2016.01.006

[10] Kang, J., Kim, K., Lim, Y. M., and Bolander, J. E. 2014. Modeling of FiberReinforced Cement Composites: Discrete Representation of Fiber Pullout. International Journal of Solids and Structures 
51:1970-1979.

\section{doi:10.1016/j.ijsolstr.2014.02.006}

[11] Naaman, A. E., Namur, G. G., Alwan, J. M., and Najm, H. S. 1991. Fiber Pullout and Bond Slip. I: Analytical Study. Journal of Structural Engineering 117:2769-2790. doi:10.1061/(ASCE)07339445(1991)117:9(2769)

[12] Kang, J., and Bolander, J. E. 2015. Multiscale Modeling of Strain-Hardening Cementitious Composites. Mechanics Research Communications In Press. doi:10.1016/j.mechrescom.2015.08.004

[13] Kawai, T. 1978. New discrete models and their application to seismic response analysis of structures. Nuclear Engineering and Design 48:207-229.

doi:10.1016/0029-5493(78)90217-0

[14] Bolander, J. E., and Saito, S. 1998. Fracture Analyses using Spring Networks with Random Geometry. Engineering Fracture Mechanics 61:569-591. doi:10.1016/S0013-7944(98)00069-1

[15] Asahina, D., Ito, K., Houseworth, J.E., Birkholzer, J.T., and Bolander, J.E. (2015). Simulating the Poisson effect in lattice models of elastic continua, Computers and Geotechnics 70: 60-67. doi:10.1016/j.compgeo.2015.07.013

[16] Vořechovský, M. 2008. Simulation of simply cross correlated random fields by series expansion methods, Structural Safety, 30: $337-363$.
[17] Kang, J., Rypl, R., and Bolander, J. E. 2016. Lattice modeling of strainhardening cement composites with spatially correlated random fiber distributions. In Banthia, N. et al. (Eds.), BEFIB 2016 - 9th RILEM International Symposium on Fiber Reinforced Concrete; (under review).

[18] Herrmann, H.J. and Roux, S. (Eds.) 1990. Statistical Models for the Fracture of Disordered Media. Elsevier/North Holland, Amsterdam, The Netherlands, 353 pp. doi:10.1002/crat.2170260821

[19] Schlangen, E. and van Mier, J.G.M. 1992. Experimental and Numerical Analysis of Micromechanisms of Fracture of CementBased Composites. Cement and Concrete Composites 14: 105-118. doi:10.1016/0958-9465(92)90004-F

[20] Adendorff, C. J., Boshoff, W. P., and van Zijl, G.P.A.G. 2010. Characterisation of Crack Distribution of Strain-Hardening Cement Composites (SHCC) Under Imposed Strain. In Stellenbosch, van Zijl, G.P.A.G., and Boshoff, W. P. (eds) Proc. Int. Conf. Advances in Cement-Based Materials; pp. 215-221.

doi:10.1201/b10162-32

[21] Lee, Y., Kang, S.-T., and Kim, J.-K. 2010. Pullout behavior of inclined steel fiber in an ultra-high strength cementitious matrix. Constr. Build. Mater 24 (10): 2030 2041. doi:10.1016/j.conbuildmat.2010.03.009 